EDITORIAL

\section{Mandatory Immunization of Health Care Providers}

\author{
The Time Has Come
}

\author{
Ian Gemmill, MD, CCFP, FRCPC
}

$\mathrm{I}$ $\mathrm{n}$ this issue of the $\mathrm{CJPH}$, two articles advise on how to improve professional and public confidence in and compliance with vaccine programs. In the first, Gallant et al. ${ }^{1}$ advocate that a multiple intervention approach be used to increase the uptake of influenza vaccine among health care providers (HCPs). In the second, Wilson et al. ${ }^{2}$ make recommendations on increasing public confidence in the safety, and consequently the public acceptance, of paediatric immunization programs. Both of these articles have implications for an issue that needs debate and resolution in Canada: mandatory immunization of HCPs against influenza to protect from serious illness patients of treatment facilities and residents of long-term care facilities, many of whom are highly vulnerable and who trust that the health system will improve, not jeopardize, their health.

Past generations of HCPs have cared for people suffering not from chronic, uncommunicable diseases, but rather from infectious diseases that put these HCPs and their families at risk. Health professionals who practised in the era before immunization controlled many infectious diseases saw it as their professional duty to provide care, notwithstanding the risk. Today, by contrast, not only do $40-75 \%$ of HCPs in acute care settings eschew influenza immunization, ${ }^{3}$ some also argue their personal rights when refusing a safe and effective measure that both protects their patients and confers a benefit - rather than risk to themselves.

Public health learned from the experience of tobacco use reduction that debate about competing rights plays no role when health may be jeopardized. In the same way that public health professionals argued effectively that protection from environmental tobacco smoke was simply a health issue, not a rights issue, protection from exposure to influenza virus of the most vulnerable is similarly simply an issue of health and not of competing rights. People who are ill or medically compromised expect, quite justifiably, that the system to which they turn for care should take every possible measure to ensure that as few risks as possible are posed to them while they are patients in that system. Moreover, as society's attitudes toward duty of care evolve, neither patients nor families will tolerate what may be construed as negligence should an HCP, who has failed for whatever reason to be immunized against influenza, be the source of infection that detrimentally affects a loved one. Successful medical liability litigation has been based on less compelling grounds.

There are many good reasons for all health care providers to be immunized against influenza. Not only is it intuitive that patients will be protected when HCPs are immunized with a vaccine that is effective against circulating strains, there is also evidence that it works. ${ }^{3}$ Hospitals, moreover, are now caring for people who on average are at significantly higher risk for influenza complications than was the case in decades past (e.g., increased numbers of patients with chronic heart and lung disease). Also, people with some conditions that used to involve long stays in hospitals are now discharged incredibly quickly e.g., same-day surgery patients who in the past would have been admitted, and bypass operation patients who go home in less than a week. Those left in the hospitals now are the sickest. Second, influenza vaccine is safe and effective and provides up to $90 \%$ protection

Medical Officer of Health, Kingston, Frontenac and Lennox \& Addington Public Health, Kingston, ON Correspondence: Dr. Ian Gemmill, Medical Officer of Health, Kingston, Frontenac and Lennox \& Addington Public Health, 221 Portsmouth Avenue, Kingston, ON K7M 1V5, Tel: 613-549-1232, Ext. 234, Fax: 613-549-1209, E-mail: igemmill@healthunit.on.ca against circulating strains $s^{4,5}$ for the HCP. It prevents a highly unpleasant illness that sends its victims to bed for a week, keeps them from work and other activities, and depletes physical energy even after recuperation. It also reduces the likelihood that the HCP will take influenza home to their families, including some who may be medically compromised. Third, the health system benefits when all HCPs are immunized by reducing preventable absenteeism and the risk that the system will not be able to function if too many HCPs are ill with influenza. ${ }^{6}$ There is very little downside to a fully immunized health workforce.

The reasons that some health care providers resist taking influenza vaccine need consideration. Although every vaccine at one time or another has had aspersions cast on its safety and effectiveness, influenza vaccines seem to be favourite targets. The reasons are a matter of speculation. Because influenza is such a confounding and unpredictable virus, there are years in which a mismatch occurs between the vaccine and the circulating strains. The illness that occurs, albeit usually mitigated by the vaccine, prompts critics to say that the vaccine has no value. It may be that, because the vaccine needs to be administered every year, there are more adverse events following immunization (AEFIs) than reported with other vaccines. These events are temporally but infrequently causally related. The reaction to some HCPs (paramedics in Ontario) being singled out for mandatory administration with this vaccine may have escalated the case against it. Perhaps the legacy of the swine influenza vaccine of 1976 - in which a poor vaccine was coupled with dire predictions of a pandemic that never materialized - is still part of the mythology that surrounds this vaccine. For whatever reason, influenza vaccine has more than its share of misbeliefs and mistrust about it.

Gallant quotes abysmal levels of uptake of influenza vaccine by the very people who have promised, through their professional vows, to do no harm (non nocere), and makes suggestions on how these levels of immunization can be improved through a multi-pronged approach. ${ }^{1}$ This modus operandi has proven its effectiveness in the reduction of illness and deaths from tobacco and thus, it clearly has application to 
increase the confidence of HCPs in influenza immunization. Another lesson learned from public health's experience in reducing the detrimental effect of tobacco on health, however, is that part of that multiple approach has to be regulation. It was through policies and by-laws restricting smoking in workplaces and public venues that many of the health gains on this issue were realized. The same will no doubt be true for immunization of HCPs.

The paper by Wilson provides insight into how the concerns of HCPs about influenza vaccine may be addressed. ${ }^{2}$ Although the paper discusses ways that public confidence in paediatric vaccine programs can be enhanced, the four-point strategy outlined is highly applicable if mandatory immunization of HCPs is implemented. It comprises:

- better AEFI reporting: this measure will demonstrate to HCPs that those promoting the mandatory use of influenza vaccine are also serious about ensuring that safety is comprehensively monitored;

- no-fault compensation for AEFI: persons who have an AEFI, whether it is owing to the vaccine or not, now must appeal to courts and prove fault for compensation. No one, including an HCP immunized in a mandatory program, should have to resort to this cumbersome system. A no-fault system recognizes that vaccine programs benefit all of society and that therefore all of society should share the costs when a possible AEFI occurs;

- pre-emptive strategies to identify and manage possible emerging AEFIs: the comprehensive public health response to oculo-respiratory syndrome is a model that can be expanded to help vaccine recipients - in this case, HCPs - to feel more confident in the vaccine's safety if influenza immunization is mandatory;

- improving communication about vaccine safety and its monitoring: this may be the most important recommendation of all, as it appears that myths about influenza vaccine may be the biggest bar- rier to its acceptance. If the vaccine is mandatory, copious reliable and accurate information before and during the program is essential to its acceptance.

Gallant is right that "a multiple intervention approach provides a practical, innovative way to guide a comprehensive program to address HCP vaccination uptake needs", but it does not go far enough. Despite efforts for years by dedicated prevention advocates to encourage all HCPs to be immunized, levels remain unconscionably low. ${ }^{3}$ It is worthwhile to pursue Gallant's strategy in the short term to ascertain whether gains can be made through that approach. At the same time, however, because many HCPs have not fulfilled their professional obligation by taking this vaccine voluntarily, the process to make it mandatory should be initiated as well. The National Advisory Committee on Immunization (NACI) has made strong statements about professional duty, ${ }^{5}$ and other professional organizations (the Association of Local Public Health Agencies in Ontario) fully support this recommendation.

The educative and regulatory approaches are not incompatible; in fact, education can assist the regulatory approach significantly. Furthermore, the suggestions made by Wilson provide additional ancillary measures that are needed for better professional confidence and that demonstrate a true commitment to monitoring safety and dealing with any real or temporally associated adverse events following immunization. All of these recommendations should be undertaken immediately so that the ultimate goal of reducing preventable exposure of the most vulnerable to a serious infectious disease can be achieved before another preventable outbreak leads to unnecessary deaths, unnecessary outbreak control measures and costs, unnecessary investigations into the professional conduct of HCPs, and unnecessary worry and anxiety on the part of all concerned.

Clearly, a safe vaccine that is effective against all influenza viruses, rather than subspecies, and that has a longer duration of protection is the ultimate goal for influenza control programs. When such a vaccine is available, the efforts to immunize millions of Canadians annually will be unnecessary and higher sustained levels of HCP immunization may be achievable through a voluntary program. In the meantime, public health professionals should advocate mandatory use of this vaccine for HCPs, the multi-faceted approach to promote and increase the use of this vaccine among HCPs described by Gallant, and the mechanisms to increase the acceptance of this vaccine among HCPs outlined by Wilson. They should also be the first to reach $100 \%$ compliance with influenza immunization, to demonstrate their commitment to preventing the unnecessary harm caused by this disease.

The sooner that routine use of influenza vaccine is comprehensively implemented, the better. A voluntary program with full compliance would be highly desirable, but in its absence, public health must lend strong support to the mandatory approach for the protection of those who expect their health care providers and the health system to heal them, and not to make them ill.

\section{REFERENCES/RÉFÉRENCES}

1. Pierrynowski Gallant DM, Murray MA, McNeil S. Influenza vaccination: A call for a multiple intervention approach. Can J Public Health 2006;97(2):136-38

2. Wilson K, Barakat M, Mills E, Ritvo P, Boon H, Vohra S, et al. Addressing the emergence of pediatric vaccination concerns: Recommendations from a Canadian policy analysis. Can J Public Health 2006;97(2):139-41.

3. Potter J. Influenza vaccination of healthcare workers in long-term-care hospitals reduces the mortality of elderly patients. I Infect Dis 1997; 175:1-6.

4. Health Canada. Canadian Immunization Guide. 2002;122-27.

5. Orr P. Statement on Influenza Vaccination for the 2004-05 Season. CCDR 2004 ACS-3:1-32.

6. Nichol KL. Influenza vaccination of healthcare workers. Infect Control Hosp Epidemiol 1997;18:189-94.

7. Wilde JA. Effectiveness of influenza vaccine in health care professionals. JAMA 1999;281:90813.

8. Nguyen-Van-Tam J. Do influenza epidemics affect patterns of sickness absence among British hospital staff. Infect Control Hosp Epidemiol 1999;20:691-94. 
ÉDITORIAL

Immunisation obligatoire des
dispensateurs de soins de santé

\author{
L'heure est venue
}

Ian Gemmill, MD, CCFP, FRCPC

$\mathrm{D}$

ans ce numéro de la RCSP, deux articles proposent des solutions pour améliorer la confiance des professionnels et du public envers les programmes de vaccins et la conformité à ces programmes. Dans le premier article, Gallant et al. ${ }^{1}$ préconisent une approche axée sur des interventions multiples pour accroître l'acceptation du vaccin antigrippal par les dispensateurs de soins de santé (DSS). Dans le second, Wilson et al. ${ }^{2}$ recommandent des solutions pour convaincre le public de l'innocuité des programmes d'immunisation pédiatrique, et donc pour mieux les faire accepter. Ces deux articles ont des conséquences sur un enjeu qui reste à débattre et à résoudre au Canada : l'immunisation obligatoire des DSS contre la grippe pour empêcher que les patients des établissements de santé et les résidents d'établissements de soins de longue durée ne tombent gravement malades. Ces personnes, dont beaucoup sont déjà très vulnérables, s'attendent en effet à ce que le système de soins améliore leur santé, et non le contraire.

Les générations passées de dispensateurs de soins de santé s'occupaient de personnes qui ne souffraient pas de maladies chroniques non transmissibles, mais plutôt de maladies infectieuses dangereuses pour ces DSS et leurs familles. Les professionnels de la santé qui travaillaient avant que l'immunisation ne permette de maîtriser de nombreuses maladies infectieuses considéraient comme un devoir professionnel de fournir des soins, quel qu'en soit le risque. Aujourd'hui par contre, non seulement de 40 à $75 \%$ des DSS qui travaillent dans des établissements de soins actifs s'abstiennent-ils de se faire immuniser contre la grippe $^{3}$, mais certains invoquent leurs droits individuels lorsqu'ils refusent une mesure sûre et efficace qui protégerait leurs patients tout en présentant un avantage - et non un risque - pour leur propre personne.

L'expérience de la lutte antitabac nous a appris que le débat sur les droits concurrents n'a pas sa place lorsque la santé peut être compromise. Les professionnels de la santé publique ont réussi à faire valoir que la protection contre la fumée secondaire du tabac est strictement une question de santé, et non de droits. Il en va de même pour la protection contre l'exposition des personnes les plus vulnérables au virus de la grippe, qui n'est pas non plus une question de droits concurrents. Les personnes malades ou immunodéprimées s'attendent avec raison à ce que le système vers lequel elles se tournent pour être soignées prenne toutes les mesures possibles pour ne pas les exposer à des risques lorsqu'elles sont entre ses mains. De plus, les attitudes sociétales face au devoir de soigner évoluent, et ni les patients, ni leurs familles ne toléreront ce qui pourrait être interprété comme de la négligence. Ce pourrait être le cas si un DSS ne se fait pas vacciner contre la grippe, quelle qu'en soit la raison, et cause une infection qui compromet la santé d'un être cher. Des procès en responsabilité médicale ont été gagnés pour bien moins que cela.

Il y a beaucoup de bonnes raisons pour tous les dispensateurs de soins de santé d'être vaccinés contre la grippe. Les patients sont bel et bien protégés lorsque les DSS sont immunisés par un vaccin efficace contre les souches en circulation - la preuve en est faite ${ }^{3}$. Qui plus est, les hôpitaux s'occupent maintenant de personnes qui, en moyenne, présentent un risque sensiblement plus élevé de complications de la grippe que ce n'était le cas au cours des dernières décennies (p. ex., un nombre accru de patients ayant des cardiopathies ou des pneumopathies

Médecin-hygiéniste de la circonscription sanitaire de Kingston, Frontenac et Lennox et Addington (Ontario)

Correspondance : Dr lan Gemmill, médecin-hygiéniste, Circonscription sanitaire de Kingston, Frontenac et Lennox et Addington, 221 Portsmouth Avenue, Kingston (Ontario) K7M 1V5. Tél. : (613) 549-1232, poste 234; téléc. : (613) 549-1209; courriel : igemmill@healthunit.on.ca chroniques). Et des malades qui autrefois auraient été hospitalisés un certain temps reçoivent maintenant leur congé avec une rapidité époustouflante; c'est le cas des patients des chirurgies d'un jour, qui auraient été hospitalisés par le passé, et des patients qui subissent un pontage coronarien, qui sont de retour chez eux en moins d'une semaine. De nos jours, ceux qui restent dans les hôpitaux sont les plus malades. Deuxièmement, le vaccin antigrippal est sûr et efficace, et il offre aux DSS une protection pouvant aller jusqu'à $90 \%$ contre les souches en circulation ${ }^{4,5}$. Il permet d'éviter une maladie très désagréable qui tient ses victimes alitées pendant une semaine, les empêche de travailler ou de pratiquer d'autres activités et les laisse physiquement épuisées même après leur rétablissement. Le vaccin réduit aussi la probabilité qu'un DSS ne propage la grippe dans sa propre famille, y compris à des proches immunodéprimés. Troisièmement, quand tous les DSS seront vaccinés, le système de santé profitera d'une baisse de l'absentéisme évitable et craindra moins d'être paralysé par l'absence d'un trop grand nombre d'employés grippés ${ }^{6}$. La vaccination générale des effectifs de santé comporte en fait très peu d'inconvénients.

Les raisons pour lesquelles certains dispensateurs de soins de santé ne se font pas vacciner contre la grippe méritent d'être étudiées plus avant. L'innocuité et l'efficacité de tous les vaccins sont mises en doute à un moment ou à un autre, mais les vaccins antigrippaux semblent être les cibles favorites de telles rumeurs. On ne sait pas vraiment pourquoi. Il est vrai que le virus de la grippe est si déconcertant et si imprévisible que certaines années, le vaccin ne correspond pas aux souches en circulation. La maladie qui survient, même si elle est en général atténuée par le vaccin, encourage les critiques à dire que le vaccin est inutile. Comme le vaccin doit être administré chaque année, on rapporte davantage de manifestations postvaccinales indésirables que pour les autres vaccins ce pourrait être une explication. Ces manifestations, reliées au moment de l'administration du vaccin, sont cependant rarement causées par ce dernier. Le fait que certains DSS (des ambulanciers paramédicaux en Ontario) ont été forcés de recevoir le vaccin pourrait avoir donné des munitions au mouvement antivaccins. 
Peut-être aussi que l'affaire du vaccin contre la grippe porcine de 1976 - on avait administré un vaccin de piètre qualité pour contrer des prédictions sinistres de pandémie, laquelle ne s'est jamais produite - a laissé des traces dans la mythologie qui entoure le vaccin antigrippal. Quelle qu'en soit la raison, ce vaccin est source de méfiance et suscite des faussetés dans une proportion démesurée.

Gallant cite les niveaux extrêmement bas d'acceptation du vaccin antigrippal chez des personnes qui, paradoxalement, ont juré en prononçant leurs vœux professionnels de ne pas nuire (non nocere); son article suggère des moyens de relever ces niveaux d'immunisation par des interventions multiples ${ }^{1}$. L'efficacité d'une telle approche a été prouvée lorsque les maladies et les décès dus au tabac ont diminué, et elle pourrait de toute évidence contribuer à accroître la confiance des DSS envers l'immunisation contre la grippe. La santé publique a cependant tiré une autre leçon de son action pour démontrer l'effet nuisible du tabac pour la santé : la réglementation doit être l'une des interventions envisagées. C'est grâce à des politiques et à des règlements limitant le tabagisme en milieu de travail et dans les lieux publics que l'on a réalisé bon nombre de gains pour la santé. Il en ira sans doute de même pour l'immunisation des DSS.

L'article de Wilson indique des moyens possibles d'atténuer les craintes des dispensateurs de soins de santé à l'égard du vaccin antigrippal ${ }^{2}$. On y décrit les moyens d'améliorer la confiance du public envers les programmes de vaccins pédiatriques, mais la stratégie en quatre points esquissée dans l'article s'appliquerait très bien si l'immunisation obligatoire des DSS était mise en œuvre. En voici les grandes lignes :

- Une meilleure notification des manifestations postvaccinales indésirables (MAPI) : cette mesure prouvera aux DSS que les tenants du vaccin antigrippal obligatoire sont par ailleurs résolus à assurer une surveillance globale de son innocuité;

- L'indemnisation sans égard à la responsabilité en cas de MAPI : pour être indemnisées, les personnes qui présentent une MAPI, que ce soit en réaction au vaccin ou non, doivent pour l'instant se tourner vers les tribunaux et prouver que le vaccin était responsable de la manifestation indésirable. Personne, à plus forte raison un DSS vacciné dans le cadre d'un programme obligatoire, ne devrait avoir à recourir à un système aussi lourd. L'indemnisation sans égard à la responsabilité reconnaîtrait que les programmes de vaccins profitent la société entière, et donc que la société entière doit en partager les coûts en cas de MAPI;

- Des stratégies préventives pour déceler et gérer les nouvelles MAPI possibles : la réaction générale de la santé publique face au syndrome oculo-respiratoire est un modèle que l'on pourrait étendre pour aider les personnes vaccinées - ici, les DSS - à être plus fermement convaincues de l'innocuité du vaccin si l'immunisation contre la grippe était obligatoire;

- L'amélioration des communications sur l'innocuité et la surveillance du vaccin : c'est peut-être la recommandation la plus importante de toutes, car il semble que les mythes qui entourent le vaccin antigrippal sont le principal obstacle à son acceptation. Si le vaccin devient obligatoire, il faudra absolument, pour le faire accepter, véhiculer une masse d'informations fiables et exactes à son sujet, avant et pendant le programme.

Gallant a raison de dire qu' "une approche axée sur des interventions multiples serait un moyen concret et novateur d'orienter un programme global visant à faire accepter le vaccin par les DSS ", mais il faut aller plus loin. Malgré les années d'efforts de partisans de la prévention dévoués pour encourager tous les DSS à se faire vacciner, leurs niveaux d'immunisation demeurent scandaleusement faibles ${ }^{3}$. Il vaudrait la peine d'adopter la stratégie de Gallant, dans l'immédiat, pour établir avec précision s'il est possible de faire des gains par cette approche, mais en même temps, puisque de nombreux DSS ne respectent pas leurs obligations professionnelles en se faisant vacciner volontairement, il faut amorcer un processus pour rendre le vaccin obligatoire. Le Comité consultatif national de l'immunisation (CCNI) a émis de solides déclarations sur le devoir professionnel ${ }^{5}$, et d'autres organismes professionnels (dont l'Association of Local Public Health Agencies de l'Ontario) appuient entièrement cette recommandation.

Les approches éducative et réglementaire ne sont pas incompatibles; au contraire, la première peut beaucoup contribuer à la seconde. Au demeurant, les suggestions de Wilson comportent des mesures complémentaires qu'il faudrait adopter pour rehausser la confiance des professionnels et leur prouver que l'on veut vraiment surveiller l'innocuité des vaccins et indemniser les victimes de toute manifestation postvaccinale indésirable, qu'elle soit réelle ou associée au moment de l'administration du vaccin. Toutes ces recommandations devraient être mises en œuvre immédiatement pour atteindre l'objectif ultime de réduire les expositions évitables des personnes les plus vulnérables à une maladie infectieuse grave - avant qu'une autre flambée n'entraîne inutilement des décès, des mesures et des coûts de lutte contre l'épidémie, des enquêtes sur la conduite professionnelle des DSS, et de l'inquiétude et de l'angoisse chez tous les intéressés.

De toute évidence, les programmes de lutte antigrippale cherchent en bout de ligne un vaccin sûr et efficace contre tous les virus de la grippe (et non simplement contre leurs sous-espèces), et un vaccin dont la durée de protection serait plus longue. Lorsqu'un tel vaccin sera disponible, il deviendra inutile de vacciner des millions de Canadiens chaque année, et l'on pourrait même obtenir des niveaux élevés d'immunisation des DSS grâce à un programme volontaire. Entre-temps, les professionnels de la santé publique devraient préconiser : a) l'administration obligatoire $\mathrm{du}$ vaccin aux DSS; b) l'approche multidimensionnelle décrite par Gallant pour promouvoir et accroître l'utilisation du vaccin chez les DSS; et c) les mécanismes proposés par Wilson pour mieux faire accepter le vaccin. Ils devraient aussi être les premiers à atteindre un seuil de conformité de $100 \%$ au vaccin antigrippal pour prouver leur engagement envers la prévention des méfaits inutilement causés par la grippe.

Pour ce qui est de la mise en œuvre globale de l'immunisation systématique contre la grippe, le plus tôt sera le mieux. L'idéal serait d'avoir un programme volontaire auquel tous se plieraient, mais comme ce n'est pas le cas, la santé publique doit solidement appuyer l'approche obligatoire pour protéger ceux et celles qui s'attendent à ce que leurs dispensateurs de soins de santé et le système de santé les guérissent, et non qu'ils les rendent malades.

[Les références se trouvent à page 87] 